\title{
Personalized hypertension management based on serial assessment and telemedicine: a cluster randomize controlled trial protocol in Anhui, China
}

\section{Siyi Xiao}

School of Health Service Management

\section{Xingrong Shen}

School of Health Service Management

Rong Liu

School of Health Service Management

Guixian Tong

Division of Medical Engineering

Tongzhu Liu

Division of Medical Engineering

Debin Wang ( $\sim$ dbwang@vip.sina.com )

Anhui Medical University https://orcid.org/0000-0002-5106-6427

\section{Protocol}

Keywords: Cluster randomized controlled trial, hypertension, behavioral intervention, personalized management, China

Posted Date: August 4th, 2020

DOI: https://doi.org/10.21203/rs.3.rs-50550/v1

License: (c) (i) This work is licensed under a Creative Commons Attribution 4.0 International License. Read Full License 


\section{Abstract}

Background: Despite tremendous investment worldwide, hypertension treatment and control rates remain low, suggesting that contemporary population-based hypertension management may have reached its efficacy ceiling. The complexity and long-term dynamics of influencing factors make personalized management inevitable and challenging. This protocol describes Personalized Hypertension Management in Anhui, China (PHMA), a project that uses a package of innovative approaches in tailoring interventions to individual patient's dynamic complications and contexts.

Methods/design: PHMA strives to reduce hypertension harms by eight proven "objective behaviors" (e.g., self-monitoring and reporting, healthy diet, physical exercise/activities). These objective behaviors are promoted through five intervention measures: support for self- monitoring, supervised machine communications, daily education or reminder messages, weekly blood pressure notification, and quarterly signed feedback. PHMA uses ten categories and over 300 variables in selecting and refining intervention procedures and content for individual patients. Efficacy of the intervention package is evaluated using a cluster randomized controlled trial design involving a total of 60 site communities and 3352 hypertension patients. Primary measure for the evaluation is systolic and diastolic blood pressure; while secondary evaluation measures include quality of life (EQ5D-5L), occurrence of hypertension-related complications (such as cerebral hemorrhage, coronary heart disease, myocardial or cerebral infarction), healthcare utilization and scores of objective behaviors.

Discussion: PHMA uses novel, low cost and sustainable approaches to tailor interventions to the dynamic conditions and contexts of individual patients. Unlike contemporary approaches to hypertension management which are mainly population based, each participant patient in PHMA applies a unique intervention package and all messages, feedbacks and other materials sent out to individual patients are different from each other. PHMA is the first project that adopts so comprehensive tailoring and if proved effective, it should have important implications for future research, practice and policy-making.

Trial registration: ISRCTN10999269. July 17, 2020; https://doi.org/10.1186/ISRCTN10999269

\section{Background}

The number of patients with hypertension has reached 1.13 billion worldwide [1]. It has increased by $30.0 \%$ since 1990 and it is expected to increase to 1.56 billion by 2025 [2]. Hypertension causes heavy disease and economic burden to patient and society. It is the major risk factor of stroke, heart disease, renal dysfunction and other important diseases [3] and causes an annual deaths of about 9.4 million ( $17.8 \%$ of the total deaths) globally [4]. Hypertension epidemic and burden in China are equally serious. Total hypertension patients and annual deaths attributable to the disease in the country amounted to 300 million and 2.5 million respectively $[5,6]$. The nation's annual direct medical expenses due to cardiovascular diseases was estimated as 130 billion yuan (or 18.4 billion USD), of which 36.6 billion yuan (or 5.2 billion USD) was for hypertension treatment [7]. 
Human responses to hypertension can be divided into two main areas, i.e., clinical treatment and behavioral intervention. Clinical responses comprise antihypertension medication and detection and treatment of hypertension complications. Behavioral interventions include medication adherence and lifestyle modifications, e.g., healthy diet, physical exercise, alcohol and tobacco containment, stress and insomnia coping, and family engagement [8-12]. However, the effectiveness of existing efforts falls far from expected. Most published interventions demonstrated only minor to moderate efficacy $[13,14]$. According to systematic reviews published by The Lancet in 2019, the treatment and control rate in highincome countries was $60.6 \%$ and $36.8 \%$ respectively [3]; and $29.9 \%$ and $10.3 \%$ in low- and middle-income countries [15]. The treatment and control rate in China was $38.3 \%$ and $14.5 \%$ [16].

Although a variety of reasons are attributable to the low treatment and control rates, lack of adequate sensitivity to the real needs of individual patients may play a key role. Contemporary approaches to hypertension management are mainly population rather than individual oriented [17-19]. Population level initiatives are highly feasible since they use only a single protocol for all individuals but suffer from inherent inability in tailoring interventions to the heterogeneous and changing conditions and contexts of individual residents [20]. Both antihypertension treatment and behavior modifications belong to life-time endeavor needing continued persistence under complex and dynamically contexts. This complexity and long-term dynamics make personalized hypertension management (PHM) inevitable since the chance to find identical hypertension determinant systems from different patients decreases exponentially as the number of its influencing factors multiplied by the number of potential variations in each of the factors at different time points increases.

However, PHM requires: a sustained information mechanism capable of constantly collecting and preserving data about relevant status and trajectory of individual patients; a sensitive planning mechanism capable of identifying personalized needs from the serial and multisource data and designing tailored management accordingly; and an effective delivery mechanism capable of implementing the planned interventions on a patient-by-patient base. These are almost impractical with traditional or mainly manual hypertension management. Due to rapidly advancing computing and communication technologies, more and more hypertension patients are using electronic tonometer, electronic weight scale, smartphone and other mobile devices in monitoring their blood pressure (BP) and related conditions [21-23]. This protocol describes a novel PHM package in Anhui, China (hereafter referred to as PHMA) which takes advantages of these new development and incorporates practical technologies from mobile and telemedicine, cloud computing, and programmed algorithms and models in a synergetic way in tackling the PHM requirements.

\section{Aim/Objectives}

The primary goal of this study is to test the efficacy of PHMA. The study hypothesis is that hypertension patients in the intervention arm will, compared to those in the control condition, demonstrate: a) lower systolic and diastolic BP: b) higher scores on objective behaviors including self-monitoring, treatment 
adherence, healthy diet, physical activities, tobacco/alcohol containment, anxiety/insomnia coping, family engagement; and c) reduced use of medical care due to hypertension and its complications. A second objective of this study is to identify key facilitators, barriers and corresponding strategies in disseminating and implementing PHMA.

\section{Methods/design}

\section{Intervention ingredients and tailoring}

\section{Overall framework}

As shown in Figure 1, PHMA aims at preventing and reducing hypertension harms by eight objective behaviors including: a) attending and responding to project messages/ contacts; $b$ ) performing selfmonitoring and reporting; $c$ ) modifying unhealthy diet habits or practices; d) maintaining adequate physical exercise/activities; e) containing tobacco and alcohol consumption; $\mathrm{f}$ ) addressing emotion and sleep problems; g) using clinical checkups and treatment; and h) facilitating family engagement and support. These objective behaviors are promoted through two intervention stages and five intervention measures. More specifically, the intervention for each participating patient starts with an orientation stage followed by a problem-solving stage. The orientation stage aims at equipping the patient with essential knowledge and attitudes about each of the objective behaviors applicable and helping him/her start to practice these behaviors. While the problem solving stage facilitates the patient to identify and solve problems or barriers encountered in practicing the objective behaviors initiated in the orientation phase. The five intervention measures are: support for self- monitoring (11), supervised machine communication (I2), daily education or reminder message (I3), weekly blood pressure notification (14), and quarterly signed feedback (15). Design of detailed content or procedures of these measures is guided by proven theories or strategies including system synergy [24], health belief model [25], social cognition theory [26], motivational interviewing [27], nudging strategies [28] and computerized tailoring. The following subsections briefly introduce each of the intervention measures.

\section{Support for self-monitoring (I1)}

PHMA provides necessary technical support for participant patients to facilitate their practice of the objective behaviors especially self-monitoring. First, it maintains a cloud database and web-based support system capable of receiving, storing and processing patients' self-monitored data and allowing them to view their personal records and feedbacks. Second, the project provides each participant patient with an electronic tonometer which: allows the patient to measure point SBP, DBP and pulse at home; sends the results to a cloud data center maintained by the project; and receives and plays voice messages disseminated from the cloud center. Third, the project encourages the participating patients to log onto their personal online accounts to: view their hypertension management profile (Figure 2) and all the messages, notifications, feedbacks and other IEC (information, education and communication) 
materials he/she has received in the past; and administer relevant self-monitoring questionnaires (Appendix 1) as suggested.

\section{Supervised machine communication (I2)}

PHMA delivers supervised machine communication (SMC) via telephone at the beginning when the patient enrolls into the project and then at the end of every three months. The communication is conducted mainly by an Al (artificial intelligence) voice processing system according to preset transcripts but the process is supervised by a human professional to handle exceptional machine-patient interactions. SMC comprises baseline and follow-up sessions lasting for about half an hour each. The baseline SMC introduces PHMA and invites participation in the project, and, if successful, performs a brief yet comprehensive assessment of the patient's: a) diagnosis and treatment history; b) symptoms of hypertension complications; and c) BP-related lifestyle practices. Each of the follow-up SMC session focuses primarily on the patient's hypertension management in the past quarter and discusses: status and trends of his/her BP; efforts and progresses made in managing his/her hypertension; difficulties encountered; and plans or suggestions for overcoming the difficulties.

\section{Daily education or reminder message (13)}

PHMA sends short (less than 120 Chinese characters), daily (once a day), and bi-modality (text and voice) messages via: a) voice electronic tonometer; and b) WeChat (the most popular social media in China), if the patient is a frequent WeChat user; or $\mathrm{c}$ ) mobile phone, if the patient uses mobile phone but are not used to WeChat. Content of the messages differs from patient to patient and from time to time but has changing focuses depending on the actual sequence of objective behaviors to be promoted and behavior problems to be addressed. Taking the example shown in Figure 2, the "intervention focus" during weeks 16 to 32 was " $C$ " and so all messages send to that specific patient during these weeks centered on "modifying unhealthy diet". All the messages are purposefully kept short to minimize burden of reading/ listening and the "one message every day without stop" strategy is adopted to maintain a consistent bond with the patient and reinforce the objectives behaviors continuously.

\section{Weekly blood pressure notification (14)}

PHMA sends a weekly notification about trends in SBP, DBP, pulse BP and BP control rate in turn for every participating patient. In other words, if a patient receives a short notification about his/her SBP at the end of this week, he/she will receive a notification about DBP, pulse BP and BP control rate at the end of the next one, two and three week(s) respectively. Then these are repeated for every four weeks. Figure 3 uses SBP as an example and illustrates how the content of the notification is generated using to the patient's self-measured blood pressure data. 


\section{Quarterly signed feedback (15)}

PHMA disseminates quarterly feedbacks to each of its participant patients via: a) paper letter sent by postal service; b) personal webpage; and c) WeChat. Each feedback addresses a specific objective behavior first in the orientation phase or a specific barrier to implementing the objective behaviors later in the reinforcement phase. Content of the feedbacks consists five parts: a) identity and time, to indicate that the feedback is current and addressed to the specific patient named; b) BP performance, to tell the patient about his/her BP status and trend and indicate that the feedback centers on his/her BP; c) efforts made recently, to appreciate the patient's efforts and progresses in the previous time period and identify main areas for improvement; $d$ ) actions to take next, to propose feasible actions for the patient to take in next period; and e) signatures, to tell the patient that the feedback is produced and checked by authorized professionals and thus useful and reliable. Figure 4 provides an example feedback.

\section{Methods for tailoring interventions}

PHMA uses comprehensive approaches in tailoring interventions to the need and context of individual patients. First, PHMA requires that the intervention for any specific patient in any specific time period focuses on only one most important and feasible objective behavior or behavior problem determined by a priority score. This score is automatically generated by a computerized algorithm based on data accumulated from previous SMC with the patient and his/her self-monitoring. Second, all items in the IEC materials pool of PHMA are designed as templates inserted with variables and when any of them is sent to a specific patient, the variables are replaced with relevant values, text or images according to his/her actual conditions/contexts. The following is a "template" message: "\{CustomerDemographics.FulIName\}: Capping monthly salt intake is an effective way in controlling blood pressure. You have \{CustomerDemographics. FamilyMember.Number\} members living together. So, your family should consume less than \{ObjectiveBehavior HealthyDiet.MonthlyFamilySalt\} grams of salt a month in total."As indicated by " $\{$ ", this message contains three variables. When sent to a patient named "Zhang San" who have 3 family members (two adults and a child of 8 years), the message is change into: "Zhang San: Capping monthly salt intake is an effective way in controlling blood pressure. You have 3 members living together. So, your family should consume less than 180 grams of salt a month in total." Figure 5 shows the template of the quarterly signed feedbacks mentioned earlier and Figure 4 is in fact an example feedback generated from this template.

\section{Variables for characterizing patients}

PHMA maintains a growing pool of variables for characterizing individual patients. All these variables are classified, coded and computerized in a way that they can be easily: a) added to relevant IEC templates in intervention development; and b) replaced, in intervention implementation, with relevant values, text or images according available data about the specific patients under concern. By the time this paper is 
written, a total of ten categories and over 300 of variables have been computerized. Table 1 presents all the categories identified so far and example variables under each category.

\section{Study design and setting}

The study adopts a cluster randomized controlled trial (RCT) design involving a total of 60 site communities with 12 in the control arm and 48 in the intervention arm. The control arm maintains existing hypertension patient management; while the intervention arms, personalized hypertension management as described earlier. Project evaluation applies to both arms using the same data collection methods and by same field data collectors. As mentioned in the "intervention tailoring" subsection above, detailed intervention varies from patient to patient due to the personalized approach. So, the uneven distribution of site communities between the control and intervention arms was designed to enable detection of potential differences between the control arm and at least four main subgroups with different intervention ingredients in the intervention arm. The analysis and reporting of the trial will be in accordance with the CONSORT guidelines $[29,30]$.

\section{Selection and randomization of communities}

The RCT is implemented in Anhui, an inland province located in eastern China with a population of about 70 million living in 105 cities or counties. Selection of participating sites and patients uses a clustered randomization which proceeds in the following steps: 1) divides Anhui province into north, middle and south regions; 2 ) randomly select 4 cities/counties from each of the three regions; 3 ) randomly select five non-adjacent communities from each of the cities/counties selected; 4) randomly select 56 patients diagnosed with hypertension from the communities selected. The five communities selected from each cities/counties in steps 3 are randomly assigned to the control arm $(n=1)$ and intervention arm $(n=4)$. All the randomization is performed by a statistical professional from outside the project team.

\section{Calculation of hypertension patients}

The above sample size of participating patients is calculated on base of our primary intervention assessment measures, i.e., changes in systolic blood pressure (SBP). Based on our previous study results and the aim of comparing the effects of at least four subtypes of combinations of mainly intervention ingredients, we suppose: a) SBP reduction was about $5.0 \mathrm{mmHg}$ as compared between the intervention and control arms; b) standard deviation of the SBP reduction was $7.8 \mathrm{mmHg}$; c) ICC value was 0.05; and d) only one out of four patients in each intention arm meets the criteria to receive the pre-assigned subtype intervention. So to detect a possible absolute difference of $5.0 \mathrm{mmHg}$ with $90 \%$ power and alpha 0.05 , we need 228 patients in each arm. By allowing for a $20 \%$ attrition rate and a design effect of 2.45 , the total sample size is estimated as $3352(=2.45 * 228 * 5 * 1.2)$ and this translates into about 56 patients per community. 


\section{Measures and data collection}

The primary measures for assessing the efficacy of PHMA are SBP/DBP. The secondary measures include quality of life (EQ5D-5L), hypertension-related complications, healthcare utilization and objective behaviors. All these measures together with data about potential confounding variables (e.g., age, sex, education, years since hypertension diagnosis) are collected at the patients' households by trained data collectors at baseline and every 12 months after baseline. SBP/DBP is measured using a mercury sphygmomanometer in accordance with standard operation specifications [31]; while the remaining data are solicited using structured questionnaires (Additional file 1).

\section{Data analysis}

The data collected through the above randomized controlled experiment will be used to: a) compare the differences in SBP/DBP between the control groups and the intervention group as a whole and subgroups with different intervention ingredients in the intervention arm at different time points; and b) compare the differences, again between the control and intervention/sub-intervention groups, in terms of the secondary measures including quality of life, occurrence of hypertension-related complications, healthcare utilization, and scores of objective behaviors.

\section{Discussion}

PHMA introduces innovative approaches to tailor interventions to the complex and dynamic BP and related complications and contexts of individual patients. Contemporary approaches to hypertension management are mainly population-based though some studies have tried some extent of differentiated interventions, e.g., send a reminder to patients who have failed to attend an appointment [32]. PHMA uses ten categories of variables in selecting and refining intervention procedures and content for individual patients. As a result, each participant patient in PHMA applies a unique intervention package and all messages, feedbacks and other materials sent out to individual patients are different from each other. To our knowledge, PHMA is the first project that adopts so comprehensive tailoring and if proved effective, it should have important implications for future research, practice and policy-making.

PHMA adopts pragmatic strategies in securing feasibility and containing cost of interventions. It uses multiple modalities (e.g., text and sound) and venues (e.g., telephone, WeChat, Webpages, paper leaflets) in forming and disseminating intervention materials to suit various patients, especially old and illiterate ones. It strives to leverage mobile devices, self-monitoring, distant communications and computerized programs into effective, low cost, convenient and sustainable interventions. Taking the example of SMC, it may lead to a novel and cost-effective mode of interactions between patients and health professionals. Although a human professional is required to supervise the whole process of every SMC session, his/her workload is reduced to a large extent as compared with traditional patient-doctor communications. In a traditional communication, the doctor (or other human professionals) needs to complete a whole range 
of activities, e.g., reviewing the patient's status and history, forming an outline plan, and interacting with the patient; while in a SMC session, what the doctor needs to do is only to start the conversation by a brief introduction and then listen to the machine-patient interactions and add occasional queries or comments. In addition to workload reduction for the doctor, SMC may also exhibit good quality and experience for the patient, since the procedures and content are preset by an expert panel and then refined by computerized algorithms according to the actual conditions of the patient under concern.

PHMA incorporates guidance from multiple theories. As illustrated in Figure 1, design of detailed intervention content or procedures is guided by health belief model, social cognition theory, motivational interviewing, nudging strategies and system synergy. All these theories/strategies have been used successfully in a variety of populations and settings [33-35]. Theory-informed intervention design prevents omission of important aspects and thus enhances overall efficacy [34,36]. Each of the theories used in PHMA has its own strengths. Health belief model is useful in addressing behaviors driven by rational thinking. Nudging strategies applies to behaviors triggered by automatic cognitive processes [25, 28]. Motivational interviewing is keen at generating and maintaining adequate momentum to leverage sustained behavior changes. System synergy informs incorporating all intervention ingredients in a way that prevents equi-finality and maximizes cost-effectiveness [27, 35, 37].

\section{Abbreviations}

$<$ p PHMA introduces innovative approaches to tailor interventions to the complex and dynamic BP and related complications and contexts of individual patients. Contemporary approaches to hypertension management are mainly population-based though some studies have tried some extent of differentiated interventions, e.g., send a reminder to patients who have failed to attend an appointment [32]. PHMA uses ten categories of variables in selecting and refining intervention procedures and content for individual patients. As a result, each participant patient in PHMA applies a unique intervention package and all messages, feedbacks and other materials sent out to individual patients are different from each other. To our knowledge, PHMA is the first project that adopts so comprehensive tailoring and if proved effective, it should have important implications for future research, practice and policy-making. $</ p>$ $<p>$ PHMA adopts pragmatic strategies in securing feasibility and containing cost of interventions. It uses multiple modalities (e.g., text and sound) and venues (e.g., telephone, WeChat, Webpages, paper leaflets) in forming and disseminating intervention materials to suit various patients, especially old and illiterate ones. It strives to leverage mobile devices, self-monitoring, distant communications and computerized programs into effective, low cost, convenient and sustainable interventions. Taking the example of SMC, it may lead to a novel and cost-effective mode of interactions between patients and health professionals. Although a human professional is required to supervise the whole process of every SMC session, his/her workload is reduced to a large extent as compared with traditional patient-doctor communications. In a traditional communication, the doctor (or other human professionals) needs to complete a whole range of activities, e.g., reviewing the patient\&rsquo;s status and history, forming an outline plan, and interacting with the patient; while in a SMC session, what the doctor needs to do is only to start the conversation by a brief introduction and then listen to the machine-patient interactions and add 
occasional queries or comments. In addition to workload reduction for the doctor, SMC may also exhibit good quality and experience for the patient, since the procedures and content are preset by an expert panel and then refined by computerized algorithms according to the actual conditions of the patient under concern. $</ p>$ $<$ p PHMA incorporates guidance from multiple theories. As illustrated in Figure 1, design of detailed intervention content or procedures is guided by health belief model, social cognition theory, motivational interviewing, nudging strategies and system synergy. All these theories/strategies have been used successfully in a variety of populations and settings [33-35]. Theory-informed intervention design prevents omission of important aspects and thus enhances overall efficacy [34,36]. Each of the theories used in PHMA has its own strengths. Health belief model is useful in addressing behaviors driven by rational thinking. Nudging strategies applies to behaviors triggered by automatic cognitive processes [25, 28]. Motivational interviewing is keen at generating and maintaining adequate momentum to leverage sustained behavior changes. System synergy informs incorporating all intervention ingredients in a way that prevents equi-finality and maximizes cost-effectiveness $[27,35,37] .</ p>$

\section{Declarations}

\section{Author contributions}

SX and XS contributed equally in conceiving this project and drafting this manuscript. RL developed the web aid. GT and TL facilitated project implementation. DW provided expertise for overall design of the study, and revised and finalized the manuscript.

\section{Funding}

Development of the primitive protocol was supported by the Natural Science Foundation of China (grant number: 71774002). Refinement and implementation of the protocol is partly supported by, the Key research and development Projects in Anhui (Grant Number 1804b06020377) and partly by Fundamental Research Funds for the Central Universities (Grant Number WK9110000014).

\section{Availability of data and materials}

Not applicable

\section{Ethics approval and consent to participate}

The study protocol had been reviewed and approved by Anhui Medical University Biomedical Ethics Committee (ref: 20200936). Participation and withdrawal from the study were voluntary and it is required 
that all participation sign (for those who were literate) or tick (for those who were illiterate) written informed consent. Confidentiality of all informant information was strictly enforced.

\section{Consent for publication}

Not applicable

\section{Competing interests}

The authors declare that they have no competing interests.

\section{Acknowledgements}

Not applicable

\section{References}

1. World Hypertension Day 2019: World Health Organization; 2019 [Available from: https://www.who.int/news-room/events/world-hypertension-day-2019.

2. Worldwide trends in blood pressure from 1975 to 2015: a pooled analysis of 1479 population-based measurement studies with 19.1 million participants. The Lancet. 2017;389(10064):37-55.

3. Long-term and recent trends in hypertension awareness, treatment, and control in 12 high-income countries: an analysis of 123 nationally representative surveys. The Lancet. 2019;394(10199):639651.

4. A global brief on hypertension : silent killer, global public health crisis: World Health Day 2013: World Health Organization; 2013 [updated 2013. Available from: https://apps.who.int/iris/handle/10665/79059.

5. National guidelines for the management of hypertension in grassroots areas. Chinese Circulation Journal. 2017;32(11):1041-1048.

6. Forouzanfar MH, Afshin A, Alexander LT, Anderson HR, Bhutta ZA, Biryukov S, et al. Global, regional, and national comparative risk assessment of 79 behavioural, environmental and occupational, and metabolic risks or clusters of risks, 1990-2015: a systematic analysis for the Global Burden of Disease Study 2015. The Lancet. 2016;388(10053):1659-1724.

7. Gao RL. China Cardiovascular Disease Report 2014 (Part of Risk Factors for Cardiovascular Disease). China Medical News. 2015;30(16).

8. Caligiuri SPB, Pierce GN. A review of the relative efficacy of dietary, nutritional supplements, lifestyle, and drug therapies in the management of hypertension. Crit Rev Food Sci Nutr. 2017;57(16):35083527. 
9. Li SJ. Compliance and Self - efficacy of Drug Treatment in Community Hypertension Patients. Chin Gen Pract. 2017;20(S2):367-369.

10. Liu T, Liu X, LI SL, Liu GL. Impact of trans-theoretical model based home visiting on medication compliance behavior in community elderly hypertension patients. Chinese Journal of Nursing. 2016;51(5):629-634.

11. Sharman JE, Howes FS, Head GA, McGrath BP, Stowasser M, Schlaich M, et al. Home blood pressure monitoring: Australian Expert Consensus Statement. J Hypertens. 2015;33(9):1721-1728.

12. Yang TT, Tong YQ, Yin XX, Lu ZX, Gong YH. Evaluation of Morisky Medication Adherence Scale and Family APGAR Applied to Patients with Tuberculosis. Chinese Journal of Social Medicine. 2016;33(6):580-582.

13. Danaei G, Finucane MM, Lin JK, Singh GM, Paciorek CJ, Cowan MJ, et al. National, regional, and global trends in systolic blood pressure since 1980: systematic analysis of health examination surveys and epidemiological studies with 786 country-years and $5 \cdot 4$ million participants. The Lancet. 2011;377(9765):568-577.

14. Melgarejo JD, Maestre GE, Thijs L, Asayama K, Boggia J, Casiglia E, et al. Prevalence, Treatment, and Control Rates of Conventional and Ambulatory Hypertension Across 10 Populations in 3 Continents. Hypertension. 2017;70(1):50-58.

15. Geldsetzer P, Manne-Goehler J, Marcus M-E, Ebert C, Zhumadilov Z, Wesseh CS, et al. The state of hypertension care in 44 low-income and middle-income countries: a cross-sectional study of nationally representative individual-level data from $1 \cdot 1$ million adults. The Lancet. 2019;394(10199):652-662.

16. Yu DM, Li SJ, Ju LH, GuO QY, Xu XL, Fu P, et al. Status of hypertension awareness, treatment and control among adults in China in 2010 - 2012. Journal of Hygiene Research. 2019;48(6):913-918.

17. Jaffe MG, Lee GA, Young JD, Sidney S, Go AS. Improved blood pressure control associated with a large-scale hypertension program. JAMA. 2013;310(7):699-705.

18. Luders S, Schrader J, Schmieder RE, Smolka W, Wegscheider K, Bestehorn K. Improvement of hypertension management by structured physician education and feedback system: cluster randomized trial. Eur J Cardiovasc Prev Rehabil. 2010;17(3):271-279.

19. Mohan S, Jarhyan P, Ghosh S, Venkateshmurthy NS, Gupta R, Rana R, et al. UDAY: A comprehensive diabetes and hypertension prevention and management program in India. BMJ Open. 2018;8(6):e015919.

20. Fortuna RJ, Rocco TA, Freeman J, Devine M, Bisognano J, Williams GC, et al. A community-wide quality improvement initiative to improve hypertension control and reduce disparities. $\mathrm{J}$ Clin Hypertens (Greenwich). 2019;21(2):196-203.

21. Carey RM, Muntner P, Bosworth HB, Whelton PK. Prevention and Control of Hypertension: JACC Health Promotion Series. J Am Coll Cardiol. 2018;72(11):1278-1293.

22. Omboni S, Ferrari R. The Role of Telemedicine in Hypertension Management: Focus on Blood Pressure Telemonitoring. Curr Hypertens Rep. 2015;17(4). 
23. Silva BM, Rodrigues JJ, de la Torre Diez I, Lopez-Coronado M, Saleem K. Mobile-health: A review of current state in 2015. J Biomed Inform. 2015;56:265-272.

24. Wu B, Arranz J, Du J, Zhou D, Traulsen A. Evolving synergetic interactions. J R Soc Interface. 2016;13(120).

25. Park D-Y. Utilizing the Health Belief Model to predicting female middle school students' behavioral intention of weight reduction by weight status. Nutr Res Pract. 2011;5(4).

26. Bandura A. Health promotion from the perspective of social cognitive theory. Psychol Health. 1998;13(4):623-649.

27. Salvo MC, Cannon-Breland ML. Motivational interviewing for medication adherence. J Am Pharm Assoc (2003). 2015;55(4):e354-361; quiz e362-353.

28. Fisher B. Richard H. Thaler and Cass R. Sunstein: Nudge: Improving Decisions About Health, Wealth, and Happiness. Environ Resource Econ. 2010;47(1):149-150.

29. Moher D, Hopewell S, Schulz KF, Montori V, Gotzsche PC, Devereaux PJ, et al. CONSORT 2010 explanation and elaboration: updated guidelines for reporting parallel group randomised trials. Int $\mathrm{J}$ Surg. 2012;10(1):28-55.

30. Schulz; KF, Altman; DG, Moher D, Group C. CONSORT 2010 Statement: Updated Guidelines for Reporting Parallel Group Randomized Trials. Ann Intern Med. 2010;152(11):726-732.

31. Hemmelgarn; BR, McAlister; FA, Grover; S, Myers; MG, McKay; DW, Bolli; P, et al. The 2006 Canadian Hypertension Education Program recommendations for the management of hypertension: Part I Blood pressure measurement, diagnosis and assessment of risk. Can J Cardiol. 2006;22(7):573-581.

32. Ashoorkhani M, Bozorgi A, Majdzadeh R, Hosseini H, Yoonessi A, Ramezankhani A, et al. Comparing the effectiveness of the BPMAP (Blood Pressure Management Application) and usual care in selfmanagement of primary hypertension and adherence to treatment in patients aged 30-60 years: study protocol for a randomized controlled trial. Trials. 2016;17(1):511.

33. Arno A, Thomas $\mathrm{S}$. The efficacy of nudge theory strategies in influencing adult dietary behaviour: a systematic review and meta-analysis. BMC Public Health. 2016;16:676.

34. Barry HE, Bedford LE, McGrattan M, Ryan C, Passmore AP, Robinson AL, et al. Improving medicines management for people with dementia in primary care: a qualitative study of healthcare professionals to develop a theory-informed intervention. BMC Health Serv Res. 2020;20(1):120.

35. Lindson-Hawley N, Thompson TP, Begh R. Motivational interviewing for smoking cessation. Cochrane Database Syst Rev. 2015(3):CD006936.

36. Ntoumanis N, Ng JYY, Prestwich A, Quested E, Hancox JE, Thøgersen-Ntoumani C, et al. A metaanalysis of self-determination theory-informed intervention studies in the health domain: effects on motivation, health behavior, physical, and psychological health. Health Psychol Rev. 2020:1-31.

37. Duan XY, Sun Y, An XL, Feng Q, Pan DH. Application effect of synergy theory-centered rehabilitation nursing on nursing of patients with peripheral facial paralysis. Biomedical Research. 2017;28(12):5300-5303. 


\section{Tables}

Table 1 Categories and examples of variables used to tailor interventions 
Demographics of patients (CustomerDemographics)

- Patient's full name (CustomerDemographics. FullName);

- Patient's Sex (CustomerDemographics.Sex);

- Patient's Family name (CustomerDemographics. FamilyName);

Status and trend of blood pressure (BPPerformance);

- Overall mean of SBP (BPPerformance.SBP.Mean.Overall);

- Mean of SBP for the last 10 times (BPPerformance.SBP..Mean.Last10Times);

- Overall trend of DBP in past year (BPPerformance.DBP.Trend.LastYear);

\section{Patient outcome (PatientOutcome)}

- Score of quality of life (EQ5D) as assessed last time (PatientOutcome.QoL.LastAssessment.Score);

- Date when quality of life was last assessed (PatientOutcome.QoL LastAssessment.Date);

- Complications occurred in the past year (PatientOutcome.Complications.LastYear. All);

Clinical history in relation to hypertension (ClinicalHistory)

- Year in which hypertension was diagnosed (ClinicalHistory.Hypertension.Diagnosis.Year);

- Whether medication treatment is in use (ClinicalHistory.Hypertension. Treatment.Current.Status);

- Type of current medications (ClinicalHistory.Hypertension.Treatment.Current.Type);

Objective behaviors (ObjectiveBehaviors)

- Title of the objective behavior scored the highest in previous assessment

(ObjectiveBehaviors.LatestAssessment. ScoredHighest.Title);

- Score of "healthy diet" in the latest assessment

(ObjectiveBehaviors.LatestAssessmen.HealthyDiet.Score);

- Title of objective behavior scored the least in latest assessment

(ObjectiveBehaviors.LatestAssessment. ScoredLeast.Title);

Problems with or barriers to objective behaviors (BehaviourProblems)

- List of problems or barriers identified from the latest assessment

(BehaviourProblems.LatestAssessment.FullList); 
- Title of problem scored the highest in the latest assessment

(BehaviourProblems.LatestAssessment. ScoredHighest.Title);

- Description of problem scored the highest in the latest assessment

(BehaviourProblems.LatestAssessment. ScoredHighest.Description);

Hypertension-related physical indicators (Physicallndicator)

- Body weight as measured last time (Physicallndicator.BodyWeight.LastMeasurement.Value);

- Date when patient body was last weighted (Physicallndicator.BodyWeight.LastMeasurement.Date);

- Waist circumference as measured last time

(Physicallndicator.WeistCircumference.LastMeasurement.Value);

Daily activities (DailyActivity)

- Common working activities as reported last time (DailyActivity.LastReport.Work.Description);

- Common working hours as reported last time (DailyActivity.LastReport.Work.Hours);

- Common leisure activities as reported last time (DailyActivity.LastReport.Leisure.Description);”

Social relations (SocialRelations)

- Number of family members (SocialRelations.FamilyMember.Number);

- Member of family members (SocialRelations.FamilyMember.RelationList);

- Family member in closest contact with the patient

(SocialRelations.FamilyMember.InClosestContact.Relations);

Resources relating to hypertension management (Resources)

- Family income per year (Resources.Faimly.Annuallncome)

- Minutes needed to get to the nearest hypertension clinic (Resources.MinutesToNearestClinic);

- Name of doctor in charge of the patient (Resources.DoctorlnCharge.Current.FullName);

Note: "()" defines the start and end of the code of the variable or variable category before it.

\section{Figures}




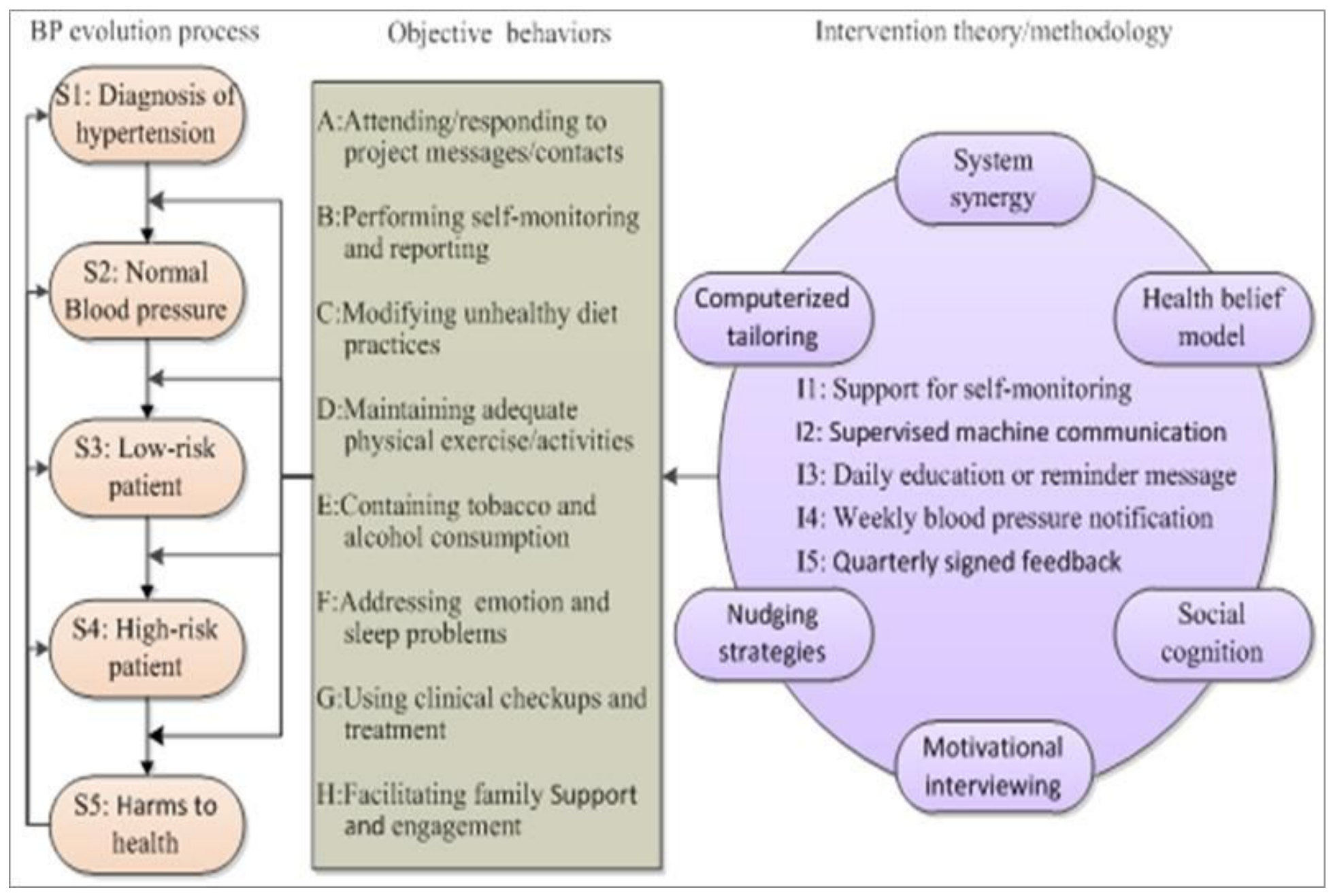

\section{Figure 1}

Conceptual framework of personalized management for hypertensive patients 


\section{Customer hypertension management profile by: March 3, 2020}

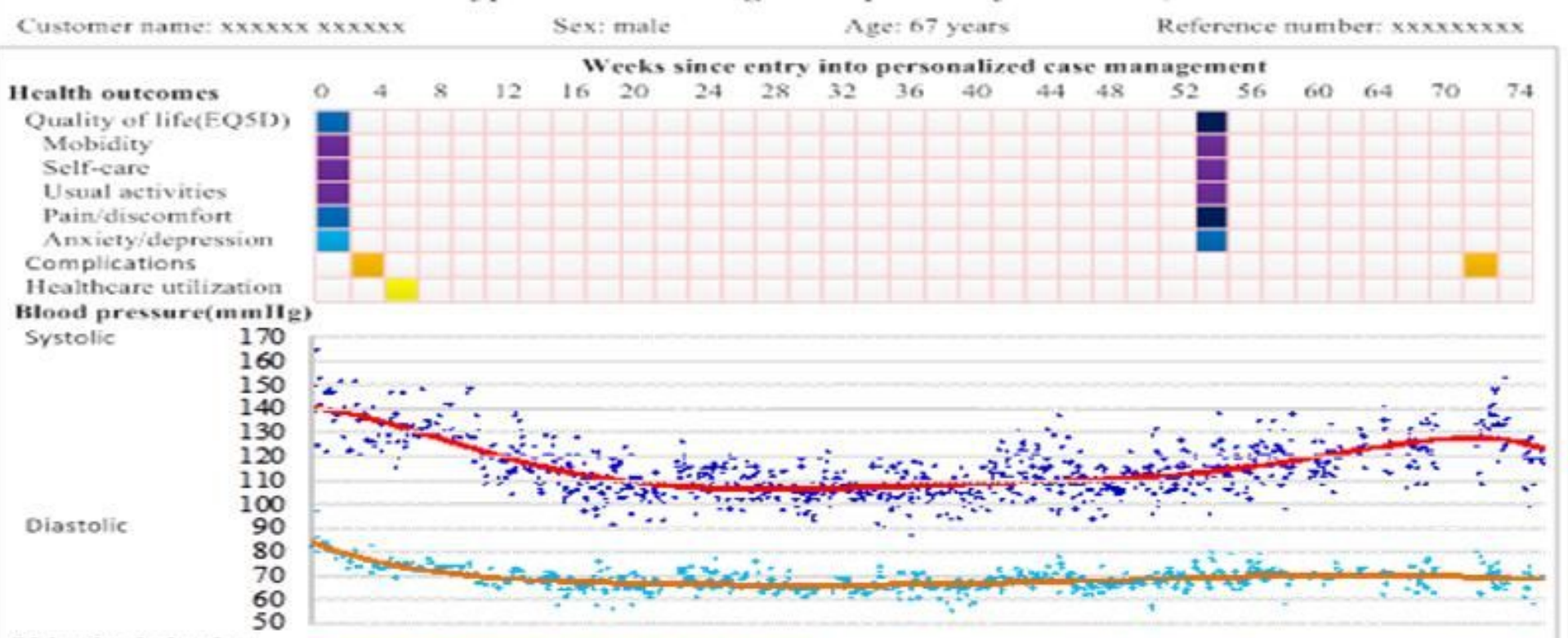

Objective behaviors

Self-monituring

Antibypertensive use

IIsalthy diet

Physical activity

Alcohol control

Tobacco control

Insomnia/mood coping

Family involvement

Intervention focuse

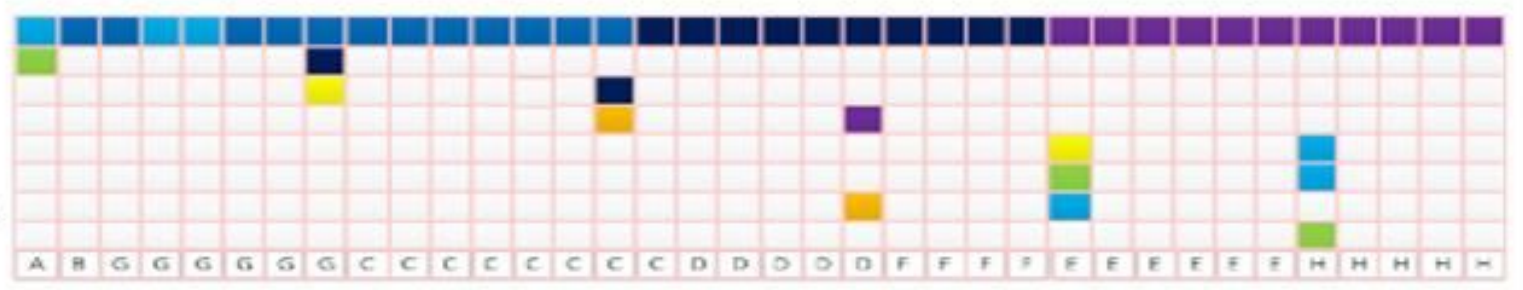

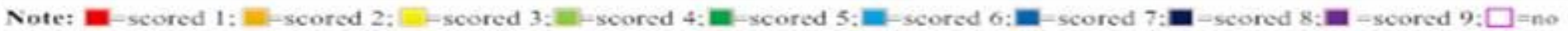

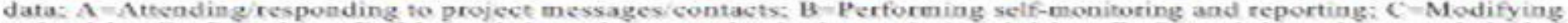

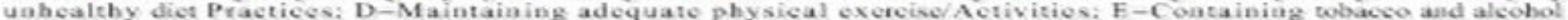

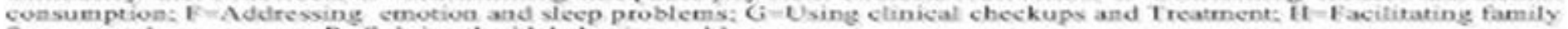
Support and engagement: $P_{1}$-Solving the ith behavior problem.

\section{Figure 2}

Example personalized hypertensive management profile 


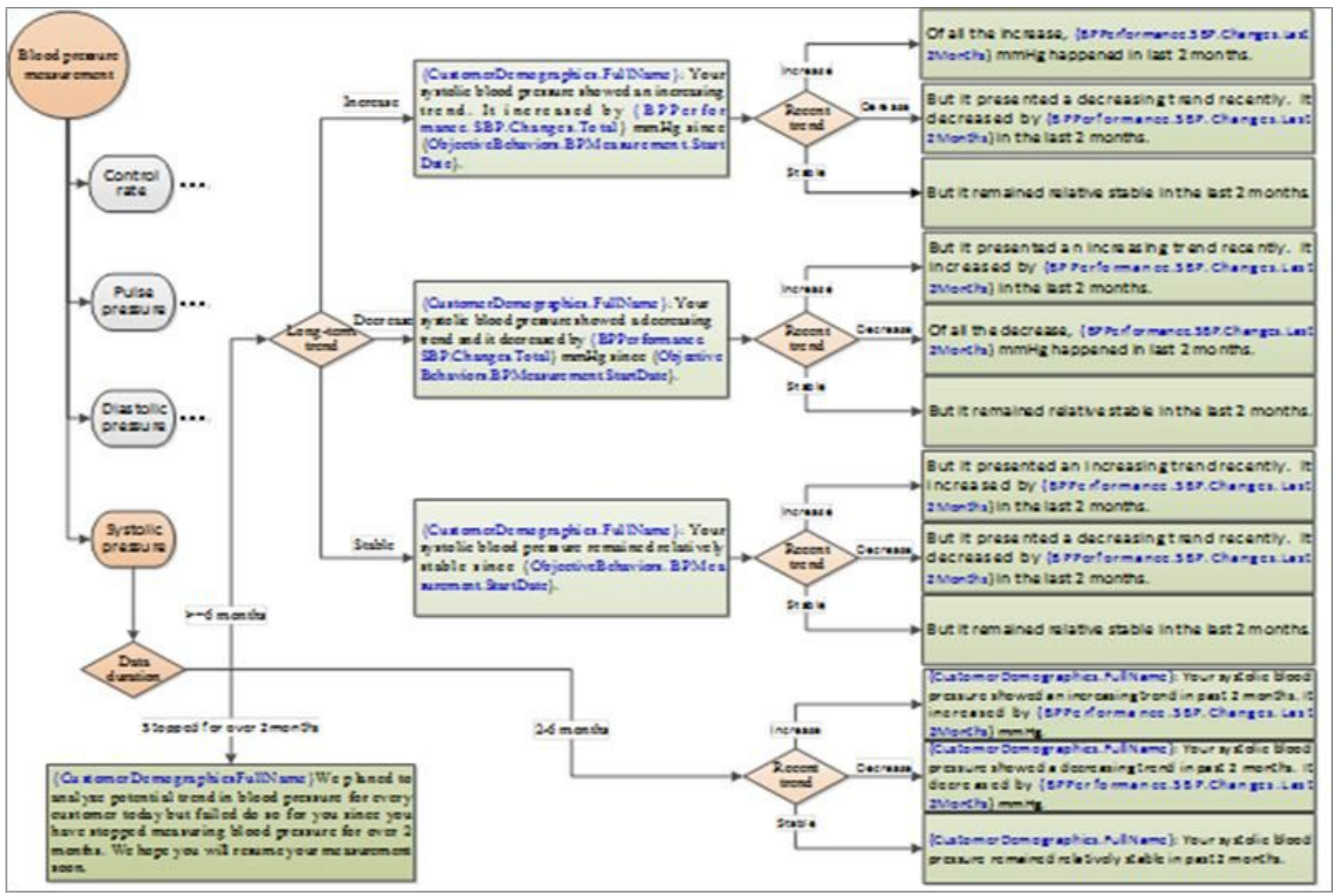

Figure 3

Example flowchart of how blood pressure notification is generated using patient self-measurements 


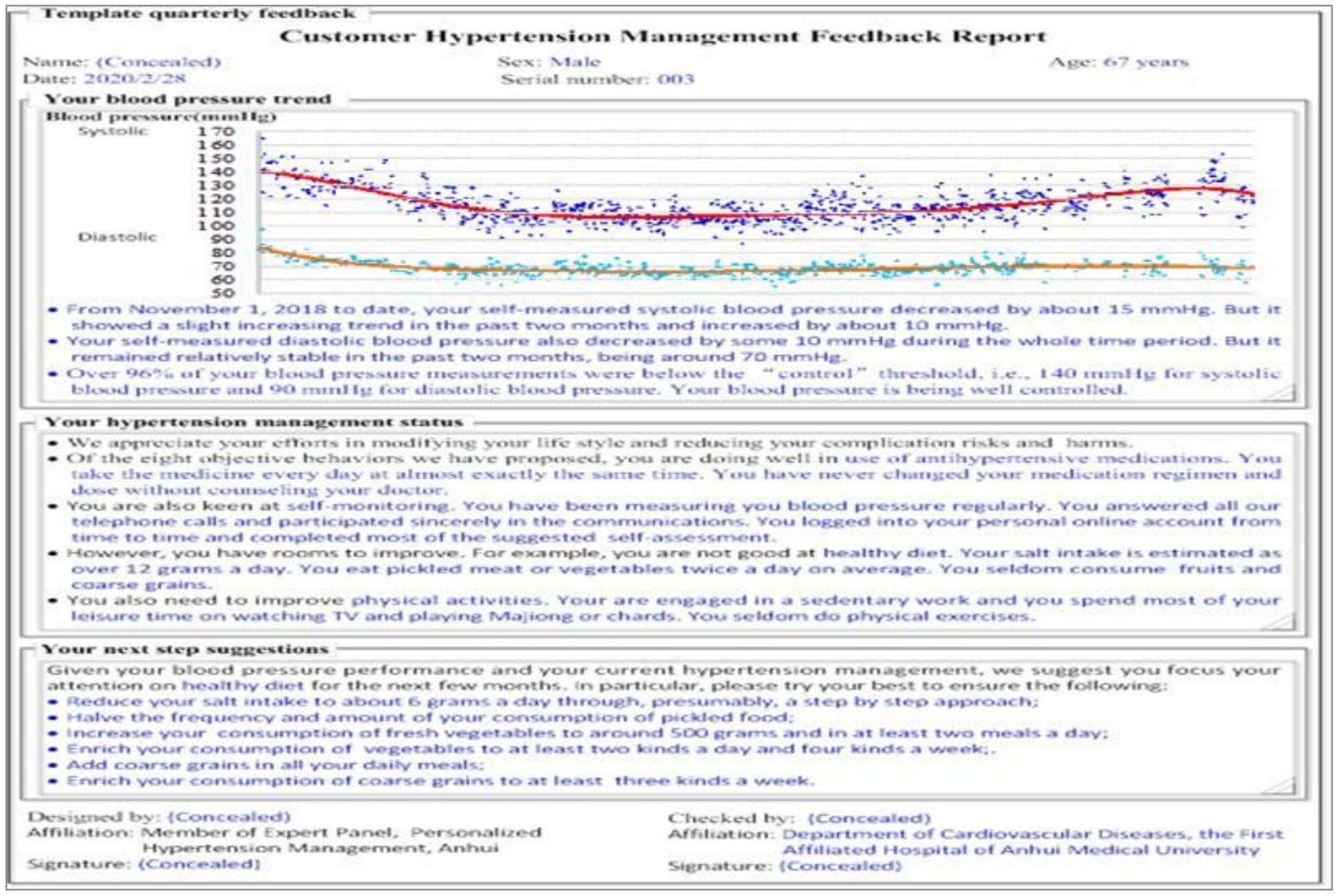

\section{Figure 4}

Example signed quarterly feedback (simplified to fit into one page) 


\section{Template quarterly feedback}

\section{Customer Hypertension Management Feedback Report}

Name: :CustomerDemographics.FullName;

Date: \{System.Date\}
Sex: CustomerDemographies.Sex:

Serial number: ;ManagementHistory. QuarterlyFeedback. Number?

Your blood pressure trend

[BPPerformance.SBP\&DBP.Scatterplot;

- \{BPPerformance.SBP.Summary\}.

- \{BPPerformance.SBP.Summary\}.

- \{BPPerformance.ControlRate.Summary\}.

\section{Your hypertension management status}

- We appreciate your efforts in modirying your life style and reducing your complication risks and harms.

- Of the eight objective behaviors we have proposed, you are doing well in fObjoctivelsehavior.Aswesament, I.astTime

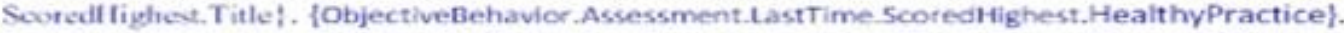

- You are also keen at \{ObjectiveBehavior.Assessment. LastTime.ScoredSecond.Title\}. \{ObjectiveBehavior.Assessment Last Time ScoredSecond.HealthyPractices\}.

- However, you have rooms to improve. For example, you are not good at (ObjectiveBehavior.Assessment.LastTime. ScoredLeast.Title). \{ObjectiveBehavior.Assessment.Last Time. SconedLeast. HarmfulPractices\}.

- You also need to improve (ObjectiveBehavior. Assessment.LastTime.ScoredSecondLeast. Title\}. (ObjectiveBehavior. Assessment.Last Time.ScoredSecondleast.HarmfulPractices).

\section{Your next step suggestions}

Given your blood pressure performance and your current hypertension management, we suggest you focus your attention on \{ObjectiveBehavior.ScoredLeast. Title\} for the next few months. In particular, please try your best to ensure the following:

- (ObjectiveBehavior.Assessment.LastTime.Scoredleast.PriorityAction1\}.

- OObjectiveBehavior.Assessment.LastTime.Scoredleast.PriorityAction2\}..

- (ObjectiveBehavior.Assessment.LastTime.Scoredleast.PriorityAction3\}..

- (ObjectiveBehavior.Assessment.LastTime.ScoredLeast.PriorityAction4\}..

- (ObjectiveBehavior.Assessment.LastTime.ScoredLeast.PriorityActionS\}..

- (ObjectiveBehavior.Assessment.LastTime.ScoredLeast.PriorityAction6\}..

Designed by: : Resourees. ExpertPanel.MemberlnCahrge.FullName; Checked by: \{Resources. DoctorinCharge. FullName; Affiliation: Member of Expert Panel, Personalized Affiliation:\{Resources.Doctorincharge.Affiliation\} Hypertension Management, Anhui

Signature: \{Resources.ExpertPanel.MemberinCahrge.Signature) Signature:(Resources.DoctorinCharge.Signature\}

\section{Figure 5}

Template signed quarterly feedback

\section{Supplementary Files}

This is a list of supplementary files associated with this preprint. Click to download.

- Additionalfile1.docx

- Additionalfile2ethicapproval.jpg 